\title{
De la respuesta correcta a la respuesta más justa. La intensidad de la tesis de la respuesta justa en las distintas etapas de la decisión judicial*
}

\author{
From the Formal Right Answer to the Justified Answer. The Intensity of Justified Answer Thesis in the Different Stages of \\ the Judicial Decision
}

Juan Bautista Etcheverry ${ }^{\text {a }}$

Universidad Austral, Argentina

jbetcheverry@austral.edu.ar

ORCID: http://orcid.org/0000-0001-9018-4293
DOI: https://doi.org/10.11144/Javeriana.vj138.drcr

Redalyc: http://www.redalyc.org/articulo.oa?id=82559799006

Fecha de recepción: 06 Febrero 2018

Fecha de aprobación: 10 Diciembre 2018

Fecha de publicación: 30 Junio 2019

\section{Resumen:}

Si bien hoy es mayoritariamente aceptado que los sistemas jurídicos resultan incapaces de ofrecer para todo caso una única respuesta correcta, todavía es posible (como hacen algunos autores) distinguir entre el plano de la corrección de una decisión jurídica de aquel de su justicia y defender que el derecho sí resultaría capaz de ofrecer un único resultado justo para todo caso. Por más que consideramos aceptable la distinción entre el plano de la corrección y el de la justicia, con este trabajo se busca mostrar que los tres principales argumentos para defender que para todo caso hay una única respuesta justa (argumento del máximo grado de concreción, de la bivalencia jurídica y de los principios) no resultan capaces de lograr su objetivo. Sin embargo, advertiremos que estos argumentos sí son capaces de mostrar que el derecho consigue con diferente intensidad acotar los márgenes de decisión judicial según en qué etapa de esa decisión nos encontremos.

Palabras clave: Única respuesta correcta, principios jurídicos, discrecionalidad judicial.

\section{Abstract:}

Although nowadays it is widely accepted that legal systems are incapable of offering a unique right answer to each case, it is still possible (as some authors do) to distinguish between the formal correction level of a legal decision and that their justice level, and to defend that law could be able to offer a justified answer to every case. As much as we consider acceptable the distinction between the plane of formal correction and the plane of justice, this paper seeks to show that the three main arguments used to defend that in every case there is a single justified answer (i.e., the maximum degree of concretion, the bivalence of legal truth and the existence of principles) are not able to achieve their objective. However, this work observes that these arguments can show that law draws limits - with different intensity - to judicial decisions depending on which stage of that decision we are.

Keywords: Right-Answer Thesis, Legal Principles, Judicial Discretion.

\section{Introducción}

Las críticas al ingenuo formalismo jurídico del siglo XIX parecían haber desterrado toda pretensión de que los jueces aplicaran mecánicamente las leyes y obtuviesen de ellas un único resultado para todo caso que se les presentara. No obstante, si bien la constitucionalización de los derechos fundamentales ha confirmado la imposibilidad de aplicar mecánicamente o de forma meramente silogística el derecho, ha resurgido el interés por establecer si el ejercicio de la función jurisdiccional presupone la existencia de una única respuesta jurídica adecuada para cada caso o, en cambio, hay cuestiones opinables sobre las que puede haber más de una respuesta que ha de ser elegida por quien decide.

Este trabajo pretende ofrecer una respuesta a este interrogante. Para ello, en primer lugar, distinguiremos las decisiones judiciales formalmente correctas de aquellas que resultan justas. Si bien en la actualidad resulta

Notas de autor:

\footnotetext{
a Autor de correspondencia. Correo electrónico: jbetcheverry@austral.edu.ar
} 
mayoritariamente aceptado que el derecho no puede aplicarse mecánicamente, ya que es posible que haya más de una respuesta formalmente correcta para un caso, todavía queda por aclararse si esto mismo sucede con las respuestas justas.

Los principales argumentos para defender la "tesis de la respuesta más justa" son tres: i) el argumento de que el máximo grado de concreción en el que se resuelven los casos exige una única respuesta; ii) el argumento de que la práctica jurídica simplifica la aplicación del derecho y la presenta como bivalente; y iii) el argumento de los principios, según el cual, entre las múltiples posibilidades interpretativas de una norma para resolver un caso en concreto, ha de decidirse por aquella que mejor realiza el conjunto de principios que justifican el derecho en general y la decisión que se ha de tomar.

Por más que se defenderá que estos argumentos no resultan capaces de conseguir su objetivo, sostendremos que sí son capaces de mostrar que el derecho logra con diferente intensidad acotar los márgenes de decisión judicial según en qué etapa de esa decisión nos encontremos.

\section{¿Hay una única respuesta correcta?}

\section{Lagunas, antinomias y textura abierta del lenguaje}

Desde mediados del siglo XIX y a medida que avanzaba el siglo XX, los teóricos del derecho han ido despertando del sueño noble de la jurisprudencia mecánica en el que muchos habían caído mecidos por el movimiento de la codificación (principalmente desarrollado en Francia) o por el de la jurisprudencia de conceptos (en Alemania) ${ }^{1}$. No obstante, el perturbador ruido de las críticas al formalismo y al logicismo jurídico terminó desvelando incluso a los más idealistas y mostrando con bastante contundencia lo ilusoria que resulta la idea de que los tribunales aplican mecánicamente las normas por medio de silogismos avalorativos de subsunción. Entre otras cosas, tales críticas han puesto de manifiesto que las normas jurídicas positivas no resultan capaces de proveer una respuesta para cada caso que se presenta - laguna- ${ }^{2}$. Frente al silencio que guarda en estos casos el derecho, el operador jurídico encargado de dirimir esta cuestión debe resolver el caso en concreto con base en argumentos o razones que van más allá de los que le proveen de una forma determinada las normas jurídicas positivas. A su vez, en algunos casos, los sistemas jurídicos acaban ofreciendo dos o más normas jurídicas positivas que pueden ser aplicadas a un caso y que llevan a soluciones incompatibles entre sí - antinomias-. Cuando las normas jurídicas parecen decir más de lo necesario para un caso en concreto y si el sistema jurídico no prevé un sistema de prelación entre esas normas, nuevamente el operador jurídico encargado de dirimir la cuestión debe hacerlo, en parte, con base en argumentos o razones que van más allá de los que le proveen las normas jurídicas positivas ${ }^{3}$. El tema de las lagunas y de las antinomias ha sido ampliamente debatido en la tradición continental europea desde hace ya más de un siglo. No obstante, en la tradición anglosajona, esta problemática ha sido abordada principalmente desde la cuestión de la "textura abierta" del lenguaje por medio del cual se expresa el Derecho. En concreto, se advierte que el lenguaje jurídico, como lenguaje humano, posee núcleos de significado claro — casos claros o paradigmáticos- en los que hay un acuerdo general sobre la aplicabilidad de un término a una realidad, y zonas de penumbra en las que se experimentan los límites de la orientación que el lenguaje general puede proporcionar. Por esto, sin importar la técnica legislativa que se utilice para comunicar los criterios generales de conducta, y por más que en muchos casos estas pautas operen sin dificultades, algún punto de su aplicación será dudoso y, por lo tanto, indeterminado ${ }^{4}$. En estos casos dudosos, las normas jurídicas positivas tienen dificultades para ofrecer una única respuesta correcta a una cuestión jurídica ${ }^{5}$. En definitiva, básicamente tres son las causas por las que inevitablemente algunas cuestiones jurídicas terminan siendo relativamente indeterminadas y, por tanto, el 
derecho no es capaz de ofrecer para todo caso una única respuesta correcta: las lagunas, las antinomias y la textura abierta del lenguaje por medio del cual se expresa el Derecho.

\section{La distinción entre el plano de la corrección y el de la justicia}

Si bien parece que el derecho resulta incapaz de ofrecer para todo caso una única respuesta correcta, todavía es posible (como hacen algunos autores) distinguir entre el plano de la corrección formal de una decisión jurídica de aquel de su justicia y defender que el derecho sí resultaría capaz de ofrecer un único resultado justo para todo caso. Así, en el plano de la corrección formal sería posible encontrar varias maneras de adecuar la conducta que se juzga al material normativo disponible y diversos modos formalmente correctos de cumplir con el precepto de una norma; esto supone admitir que hay varias respuestas formalmente correctas para un caso. No obstante, se sostiene que otra cosa sucede si se adopta la perspectiva de la justicia, de la solución justa en el caso concreto. Así, si bien desde el plano de la corrección formal puede resultar dudosa la aplicación de una norma que prohíbe el ingreso al parque con "vehículos" a una persona que por una afección física pretende hacerlo con una silla de ruedas propulsada por un motor eléctrico; no cabrían dudas desde la perspectiva de la justicia de la inaplicabilidad de tal prohibición para este caso especí fico. Tres son los principales argumentos que pueden encontrarse para defender esta posibilidad ${ }^{6}$.

\section{El argumento de los principios}

Uno de los argumentos más conocidos para defender la existencia de una única respuesta justa para cada caso pone de relieve el determinante papel que tienen los principios jurídicos a la hora de buscar una respuesta jurídica justificada que permita resolver un conflicto. En la actualidad, muchos de estos principios están expresamente recogidos por las prácticas constitucionales, legales y jurisprudenciales de los sistemas jurídicos occidentales. De hecho, hay quienes consideran que la explicitación jurídicopositiva de estos principios se va dando paulatina y conjuntamente a las lagunas, antinomias y normas vagas con las que (a pesar de lo que proponía el formalismo del siglo XIX y XX) se van enfrentando quienes han de resolver los conflictos jurídicos. Uno de los autores que con mayor repercusión defendió este argumento fue RONALD DWORKIN $^{7}$. Para este profesor estadounidense, las dificultades anteriormente mencionadas no suponen la negación de la existencia de una única respuesta jurídicamente adecuada para cada caso, si se acepta el enfoque del "Derecho como integridad". Este enfoque defiende (entre otras cosas) que aplicar una norma jurídica supone preguntarse “...qué interpretación, de las diferentes interpretaciones admisibles del significado abstracto de un término, mejor desarrollan el conjunto de principios y de políticas públicas capaces de ofrecer la mejor justificación pública para dicha norma al momento de su aprobación" ${ }^{8}$. Para DWORKIN, en esta tarea confluyen, al menos, cuatro etapas: i) preinterpretativa: en la que se identifican las exigencias jurídicas a interpretar; ii) justificacióngeneral: en la que se buscan la/s finalidad/es de todas las exigencias jurídicas; iii) encaje: en la que se busca asegurar que la justificación general identificada ofrezca una explicación aceptable de las normas e instituciones que conforman la práctica jurídica; y iv) aplicacín o reforma: se decide cómo debe aplicarse o dejar de aplicarse o reformarse una norma para que realice mejor la finalidad de la práctica ${ }^{9}$.

Sin embargo, para DWORKIN, la existencia de una única respuesta adecuada no significa que sea necesariamente la más justa para quienes la aplican. De hecho, DWORKIN realiza un esfuerzo por mostrar que su propuesta se diferencia de las visiones pragmáticas que sostienen que las decisiones políticas del pasado (como la Constitución, la legislación y los precedentes) no son capaces por sí solas de proveer ninguna justificación para el uso de la fuerza coercitiva del Estado y que, por tanto, esta justificación habría que buscarla únicamente en el valor de la justicia ${ }^{10}$. En cambio, basándose en lo que considera es la tradición 
estadounidense, propone fundar la coerción del derecho en los principios de justicia sustantiva (vinculado con la adecuada respuesta del sistema político que ha de tratar a los individuos con igual consideración y respeto ${ }^{11}$ ), equidad (entendida como la estructura que garantiza igual poder de cada uno de los individuos de una sociedad por medio de una correcta distribución del poder político; es decir, que se garantice la mayor representatividad posible en la toma de decisiones colectivas) y debido proceso (concebido como el correcto procedimiento definido representativamente para hacer valer las normas y regulaciones que el sistema jurídico produce, ya que ellas son las únicas que pueden distribuir la fuerza pública) ${ }^{12}$. A partir de estos tres principios y no solo teniendo en cuenta el de justicia sustantiva, un juez ideal debe interpretar el derecho bajo su mejor luz y construir la respuesta que mejor realiza tales principios de modo integral ${ }^{13}$.

\section{El argumento de la bivalencia de las respuestas jurdicas}

Otro argumento que suele ser presentado para defender la tesis de la única respuesta justa se basa en el hecho de que las respuestas jurídicas que los jueces han de ofrecer a los conflictos jurídicos en muchas ocasiones son presentadas como bivalentes por la propia práctica jurídica. En este sentido, los sistemas jurídicos suelen considerar que una norma es o no constitucional, que el demandado es o no responsable, que la prueba es o no válida, que el acusado es o no culpable, etc. ${ }^{14}$

Por ello, algunos autores llegan a sostener que tanto el par formado por cada uno de estos enunciados "norma constitucional", "demandado responsable", "prueba válida" o "acusado culpable" y su negación son exhaustivos respecto de toda circunstancia específica a la que cada concepto se aplica e, incluso, que lo sería cada par formado por la aplicación o la no aplicación de una norma jurídica respecto de toda circunstancia específica a la que se aplican ${ }^{15}$. Así, por ejemplo, la doctrina podrá discutir si la acción de una persona de suministrar intencionalmente vidrio molido mezclado en la comida y causar la muerte a otra persona supone cometer el delito de homicidio agravado, por ser ejecutado "con veneno" (agravante, por ejemplo, que reconoce el artículo 80 del Código Penal de Argentina). No obstante, todas las posiciones concuerdan en que o bien el vidrio molido se considera como "veneno" y, por tanto, se condena a quien cometiere un homicidio doloso con vidrio molido con una pena agravada; o bien el "vidrio molido" no se considera como "veneno" por no actuar químicamente en el cuerpo y, por tanto, no resulta aplicable al caso este tipo específico de agravante del homicidio ${ }^{16}$. En definitiva, no pudiendo ser ambas posiciones doctrinarias correctas por ser antitéticas (es decir, porque aceptar una supone negar la otra), necesariamente una sola de las respuestas acerca de si el vidrio molido debe ser considerado "veneno" será la correcta o verdadera.

\section{El argumento del máximo grado de concreción}

A su vez, también hay quienes afirman que si bien en el plano de la comprensión o de la interpretación abstracta de los principios o del resto de las normas (que siempre tienen un mayor o menor grado de generalidad o abstracción) puede existir más de una respuesta correcta, esto es poco factible en el nivel de la interpretación concreta o práctica que busca aplicar una norma a un caso concreto, en el que las conductas están máximamente determinadas y en el que resulta necesario resolverlo de modo justificado. La diferencia entre la interpretación abstracta y la concreta sería que, "[1]a interpretación concreta, típicamente desplegada a nivel judicial, formula enunciados referenciales definitivos, mediante los cuales se identifican instanciaciones concretas de los conceptos esclarecidos en la dimensión abstracta de la interpretación. Su propósito no es comparar conceptos con conceptos, sino aplicar conceptos a hechos. No pretende describir el concepto de acción voluntaria, tipos de acciones, su valor legal, y sus consecuencias, sino juzgar si los hechos concretos encajan con cada uno de estos cuatro tipos de juicios y conceptos" ${ }^{17}$. 
Para defender esta posición, se advierte que las decisiones judiciales se mueven en un ámbito de concreción en el que se daría una cumbre de determinación que no puede consistir sino en la "adopción de la mejor" o "más justa solución posible del caso concreto" ${ }^{18}$. Este argumento es defendido o respaldado principalmente por tres razones diferentes.

En primer lugar, sería así porque estas decisiones se toman por medio de un proceso que concluye en una alternativa que ha "de ser única, ya que de lo contrario no sería posible alcanzar una decisión acerca del obrar, (...) [y] si no existiera en concreto una alternativa mejor, i. e., más adecuada a la concreta determinación del bien humano, la voluntad no podría elegir su puesta en la existencia, con la resultante parálisis de toda acción humana" ${ }^{19}$. Como esa parálisis no sucede en el caso de las decisiones judiciales, entonces habría de reconocerse que para todo caso los jueces son capaces de encontrar la única respuesta que consideran adecuada. Sin embargo, esto no supone que se le niegue un papel a la voluntad que, conjugada con la inteligencia, conduciría a la alternativa mejor para el caso en concreto ${ }^{20}$.

En segundo lugar, otro grupo de autores considera que en el caso de la argumentación judicial siempre hay una respuesta adecuada porque la toma de decisiones judiciales está simplificada por el propio sistema jurídico que presenta los conflictos jurídicos concretos y determinados como bivalentes. Esto explicaría que quienes actúan en la práctica jurídica guían su comportamiento por la idea de que hay una sola respuesta, “precisamente porque la búsqueda de la misma no es 'en abstracto' (...) [sino] del caso tal y como se lo ha planteado" ${ }^{21}$. Como resulta fácil advertir, el segundo argumento para defender que en la decisión judicial concreta siempre hay una única respuesta adecuada remite al argumento de la bivalencia jurídica.

En tercer lugar, otra manera de defender el argumento del máximo grado de concreción advierte que si bien puede haber distintas concepciones acerca de la posibilidad de aplicar a un caso los conceptos con que se expresan las normas, el enfoque de la integridad permitiría llegar siempre a un único resultado jurídicamente adecuado. Dicho de otro modo: el enfoque de la integridad considera que en cada caso concreto se puede encontrar la respuesta jurídica adecuada reconstruyendo la historia del sentido de la regulación que debe aplicarse para resolver el caso, poniendo en relación tal regulación con los principios que permitirían justificar la práctica jurídica entendida como un todo y (como se explicó en el apartado sobre el argumento de principios), de este modo, conseguir criterios (razones públicas y justificadas) para determinar qué decisión o interpretación realiza mejor la práctica jurídica ${ }^{22}$.

\section{La posibilidad de que haya más de una respuesta correcta y justa}

\section{¿De qué hablamos cuando hablamos de la existencia de una única respuesta justa para cada caso?}

Antes de continuar, consideramos necesario precisar a qué se hace referencia cuando se defiende la tesis de que para cada conflicto jurídico hay "una única respuesta justa". Claramente, con esta tesis no se defiende que "la decisión judicial justa" siempre pueda ser deducida de premisas axiomáticas y evidentes. A su vez, tampoco se hace referencia a que haya una única solución justa solo porque siempre hay para cada caso una única decisión final; ya que una decisión puede ser final e injusta ${ }^{23}$. Obviamente, esta tesis tampoco se refiere a que los jueces siempre resuelvan los casos conforme a la decisión más justa; de otro modo, no habría decisiones judiciales reprochables ni tendrían sentido la multiplicidad de instancias como un mecanismo de control de la corrección de las decisiones judiciales ${ }^{24}$.

Sin embargo, no resulta claro de la defensa de la "tesis de la respuesta más justa" anteriormente expuesta si ella se aplica a todo el contenido de la decisión judicial o solamente a algunos de sus aspectos. Por ejemplo, resulta bastante dudoso que el juicio determinativo acerca de exactamente cuánto ha de pagar 
de indemnización quien ha sido juzgado como responsable por un daño causado, o el que determina específicamente el quantum de una sanción (dentro de los parámetros legales) a quien ha sido considerado culpable sean estrictamente bivalentes y conduzcan a un único resultado justo ${ }^{25}$. Por ello, a continuación explicaremos cuáles son las cuatro partes o etapas de la decisión judicial y cómo la bivalencia de las respuestas jurídicas, el razonamiento con principios y el hecho de que el fin de estas etapas sea resolver un caso concreto y no meramente conocer en abstracto el derecho vigente impactarían en cada una de esas etapas. Este esfuerzo se hace con la finalidad de poner de manifiesto que si bien resulta difícil sostener que para cada caso hay siempre una única respuesta justa que ha de ser capaz de abarcar completamente todos los aspectos de una resolución judicial; es posible, sin embargo, defender que la discrecionalidad judicial está restringida de diferente modo en cada una de las fases o partes de las decisiones judiciales.

\section{Las cuatro fases o partes de una decisión judicial}

Siguiendo a PILAR ZAMBRANO, sostendremos que el razonamiento judicial que conduce a la decisión judicial concreta puede ser dividido, al menos, en los siguientes partes o fases: la identificación de los hechos relevantes del caso que se presenta para ser resuelto de forma tal que puedan ser conceptualizados como tipos específicos de acciones; la atribución a un sujeto (o varios) como agente que realizó voluntariamente tales tipos de acciones; la identificación de las normas que regulan estos tipos de acciones y del modo en que los califican y de las consecuencias que les correlacionan; y, por último, el dictado de una norma de acción singular que establezca cómo se efectivizará la solución jurídica para el caso ${ }^{26}$.

Más detalladamente, los jueces - a fin de alcanzar una decisión capaz de resolver un caso en concreto-, por un lado, (i) han de identificar los hechos del caso que conforman o realizan un ejemplo concreto de algún tipo de acción jurídicamente contemplada. A esta etapa del razonamiento jurídico tradicionalmente se la denominó juicio de subsunción del caso en el supuesto de hecho previsto normativamente. A modo de ejemplo, puede decirse que en un caso en que alguien entrega gratuitamente a otro una cosa mueble no fungible nos encontramos frente a un contrato de comodato (cfr. artículo 1533 del Código Civil y Comercial argentino).

A su vez, (ii) los jueces deberán establecer si los hechos que generan el caso de algún modo son atribuibles a un sujeto, normalmente, en calidad de agente que obra conscientemente. Así, para que se perfeccione el comodato, será necesario no solo la entrega gratuita por parte de alguien a otro de una cosa no fungible, mueble o raíz, sino que dicha entrega sea realizada con discernimiento (es decir, por alguien en pleno uso de su razón), intención de realizar la entrega de la cosa y de facultar su uso a una persona específica (es decir, sin ignorancia o error esencial sobre la cosa, la persona o la naturaleza de la acción que se realiza) y libertad (es decir, sin mediar fuerza irresistible o intimidación capaz de causar fuerte impresión) ${ }^{27}$.

Identificados en los hechos del caso el tipo de acción y el supuesto de hecho normativo en el que podría subsumírselo y que dicha acción en principio pueda ser atribuida a un sujeto que obró voluntariamente, (iii) resta todavía verificar cómo la norma que se le aplica califica esa acción (como permitida, prohibida o no prohibida) y qué consecuencias le correlaciona a ese tipo de acciones. Por ejemplo, el Código Civil y Comercial de Argentina considera prohibido prestar una cosa (v. g., un arma de fuego) para un uso contrario a las leyes o buenas costumbres, o prestar cosas que estén fuera del comercio por nocivas al bien público y, por lo tanto, en estos casos el préstamo de uso no conformará un contrato válido (cfr. artículos 279, 281, 1003, 1004, 1012 y 1014 del Código Civil y Comercial de Argentina).

Llegados a este punto, (iv) solo faltaría formular una norma de acción singular que efectivice y, en muchos casos, determine o concrete la consecuencia jurídica que surge de las normas que se aplican para resolver el caso. Por ejemplo: el agente $\mathrm{X}$ (personal de seguridad) que recibió un arma (para su portación sin su debido permiso) por medio de un contrato de comodato de parte de $\mathrm{Y}$ (sujeto este último que además contrata los servicios de seguridad personal que ofrece X) ha de devolver esa arma en un determinado plazo específico y 
en un determinado lugar, por ser declarado dicho contrato como inválido, ya que la finalidad del préstamo es que su objeto sea utilizado con un fin (tenencia ilegítima) contrario a las leyes (cfr. artículo 189 bis del Código Penal de Argentina).

\section{La bivalencia jurídica en las cuatro etapas de la decisión judicial}

Presentadas estas cuatro etapas del razonamiento judicial, consideramos importante advertir que la bivalencia jurídica pretende lograr en diferente medida su cometido (de presentar las soluciones de los conflictos jurídicos como opciones antitéticas en las que solo una puede ser correcta, verdadera o justa) en cada una de las etapas de la decisión judicial. Más en concreto, parece que el sistema jurídico pretende una mayor bivalencia de las respuestas jurídicas en las tres primeras etapas del razonamiento jurídico que juzgan hacia atrás el actuar de una/s determinada/s persona/s, que en la cuarta etapa en las que se establece hacia delante cómo corresponde resolver los problemas de coordinación que surgieron a partir del actuar que se juzga.

Más específicamente, puede decirse que el sistema jurídico pretende un grado de precisión y previsibilidad capaz de regular anticipadamente (hacia atrás) todo conflicto de coordinación actual de modo tal que esas regulaciones del pasado puedan actuar como si proporcionaran - al momento de resolver el conflictorazones para actuar de la manera legalmente prevista. Esta pretensión se expresa por medio de técnicas como la presentación del sistema jurídico como un cuerpo normativo coherente o capaz de prever todos los casos o el uso de algunos conceptos jurídicos (llamados, por DWORKIN, "dispositivos") como si fueran bivalentes. Como hemos visto, el uso de estas técnicas es una ficciónque no ha de ser tomada literalmente. La existencia de lagunas, antinomias y la textura abierta del lenguaje por medio del cual se expresa el derecho evidencian los límites de esta pretensión y de las técnicas de las que se sirve para tal fin. Sin embargo, la existencia de esta pretensión y de tales técnicas parecen circunscribirse principalmente al razonamiento jurídico que indaga lo sucedido en un caso, y preguntarse por el tipo de acción que origina el conflicto; la posibilidad de atribuírselo (como un acto voluntario), al menos, a un sujeto; y la calificación de esa acción (como permitida, prohibida o debida) que hacen la/s norma/s que podría/n aplicársele. Así, el sistema jurídico y el uso que de él hacen los operadores jurídicos para resolver conflictos parecen pretender, por ejemplo, que resulte claro si un determinado hecho es o no un acto jurídico, si ese acto es o no un contrato, si ese contrato conforma un contrato de locación o uno de compraventa, o si una determinada persona es o no responsable por el incumplimiento de tal contrato o si resulta culpable o inocente de determinado delito, etc. ${ }^{28}$.

En cambio, el propio sistema jurídico y la práctica que de él hacen los operadores jurídicos parecen reconocer un mayor margen de maniobra para los jueces y, por tanto, una relajación de la pretensión de la bivalencia de la respuesta jurídica en los elementos de la decisión que resuelven no tanto juzgando el pasado de las acciones realizadas, sino decidiendo hacia el futuro sobre las consecuencias que les corresponden a esas acciones. Más específicamente, tanto la práctica como las normas jurídicas en muchas ocasiones ofrecen explícitamente márgenes de decisión a las consecuencias jurídicas concretas que han de aplicárseles a determinados tipos de acción previstos legalmente (v. g., "ser reprimido con prisin de 1 ao a 6 aos el que entregare un arma de fuego, por cualquier título, a quien no acreditare su condición de legítimo usuario", itálicas fuera de texto). Algo parecido sucede con los aspectos relativos a cómo concretamente han de ejecutarse las consecuencias jurídicas determinadas judicialmente para la resolución de un caso en concreto (v. g., "el señor X ha de devolver a la brevedad el arma de fuego"). Esto último es tan así que en muchos casos los jueces no especifican puntualmente cómo, cuándo y dónde se efectivizará lo decidido y dejan a las partes que lo acuerden e intervienen solo, en juicios de ejecución de sentencias, en los casos en que ellas no pudieran convenir esto o en los que una de las partes se rehusase a cumplir con lo decidido judicialmente (v. g., "el señor $X$ ha de devolver el arma de fuego en cuestión el 10 de octubre de 2017 a las 8 horas en el domicilio de su propietario..."). 


\section{El impacto del argumento de principios en las cuatro fases de la decisión judicial}

A la crítica anteriormente esbozada, podría intentar respondérsele con el argumento de los principios. Según este argumento: cuando (a) el significado abstracto del supuesto hecho o de la consecuencia jurídica vuelvan necesaria su determinación o concreción; o cuando (b) los hechos de un caso no encajen específicamente en un supuesto de hecho; o cuando (c) estos hechos encajen en más de un supuesto de hecho normativamente previsto al que se le correlacionan diferentes consecuencias jurídicas (v. g., cuando el contrato de leasing era atípico y, por tanto, no estaba claro si había que aplicársele supletoriamente las normas del contrato de compraventa o las del contrato de locación); e incluso cuando (d) resulta necesario determinar o concretar cómo ha de ser la acción singular que resuelve el caso; todo esto podrá hacerse guiados por el criterio de cuál es la opción que mejor desarrolla integralmente los principios que justifican el Derecho.

Planteado así, en definitiva, el argumento de la bivalencia (y la versión del argumento de la máxima concreción que remite al de la bivalencia) reposaría en la corrección del argumento de los principios. El problema es que este último argumento no advierte que una de las características con las que se suelen identificar muchos de los principios jurídicos (y, que solo en una medida menor resulta aplicable a las reglas) es que están intrínsecamente necesitados de concreción o determinación porque suelen admitir más de un modo similar y legítimo de ser desarrollados o efectivizados ${ }^{29}$.

Más específicamente, suele considerarse que muchos de los principios jurídicos son "abiertos", "abstractos" o "generales". En las reglas, las propiedades que conforman el caso constituyen un conjunto que pretende ser cerrado. En cambio, muchos de los principios jurídicos ni siquiera pretenden formular una lista cerrada de tales propiedades. De este modo, si se supone que en las reglas las condiciones de aplicación están concretadas y determinadas, en el caso de muchos de los principios tales condiciones apenas están muy genéricamente establecidas $^{30}$.

Todo esto genera que muchas veces la jurisprudencia que los aplica no sea espontáneamente uniforme. Por ejemplo, en la jurisprudencia argentina (especialmente en la de su Corte Suprema Nacional) se debatió ampliamente si la tenencia de estupefacientes para consumo personal podría o no ser considerada una "acción privada" (según el artículo 19 de la Constitución de Argentina) "que de ningún modo ofend[e] al orden y a la moral pública, ni perjudic[a] a un tercero" y, por lo tanto, que no podría ser punida, al estar "exenta de la autoridad de los magistrados".

Los principios jurídicos además de ser normas con condiciones de aplicación, en muchos casos, expresamente abiertas y generales, normalmente carecen de una consecuencia jurídica concreta y determinada 31. Por ello, aunque en algunos casos los principios puedan ofrecer una calificación jurídica para un tipo determinado de acción, normalmente el tipo de solución singular (remedies) que han de mandar los jueces para resolver un caso aplicando un principio continúa resultando indeterminado. Por ejemplo, si bien el artículo 17 de la Ley de Contrato de Trabajo argentina deja clara la probibición de "cualquier tipo de discriminación entre los trabajadores por motivos de sexo...", no surge de la norma qué tipo de medidas tomar en caso de que una empresa con miles de empleados solo tenga una ínfima parte de su personal de sexo femenino. En los últimos años, partiendo de este principio y otros similares (algunos incluso más generales y otros más extensos, recogidos en tratados internacionales y la propia Constitución de Argentina), la jurisprudencia argentina ha sostenido que los empleadores deben tener un "criterio neutro" en relación con el sexo del empleado a la hora de su contratación e incluso deben "rechazar aquellos otros criterios que, aun cuando sean formalmente neutros, produzcan un resultado adverso para los integrantes de uno y otro sexo". Incluso se ha llegado a condenar a una empresa a que "en el futuro, solo contrate personal femenino hasta compensar en forma equitativa y razonable la desigualdad producida" ${ }^{32}$.

Todo lo anterior impacta en el modo en que muchos de los principios jurídicos guían el razonamiento jurídico con un tipo de fuerza meramente directiva o limitada. Esto sería así, porque tales principios solo 
ofrecerían algunos de los elementos que pasarán a tener en cuenta quienes abordan el proceso racional de tomar una decisión, ya que quienes han de aplicarlos en muchos casos no pueden concluir de manera razonable simplemente apelando a cualquiera de ellos. Por esto suele decirse que para aplicar este tipo de principios jurídicos es necesario ponerlos en relación con otras razones normalmente recogidas en otros principios y reglas. Así, este tipo de principios se podría caracterizar, como hace buena parte de la literatura jurídica al hablar de principios, como normas de las que se derivan calificaciones normativas prima facie. Esto significa que solo son capaces de ofrecer una pauta para el razonamiento jurídico que se volverá definitiva únicamente después de su puesta en relación o ponderación contra otras normas cuyas calificaciones normativas sean incompatibles y también tengan un carácter prima facie ${ }^{33}$.

Recapitulando, los principios en muchos casos no prevén sus situaciones de aplicación, sino que, más bien, parecen mostrar un bien o fin que ha de ser buscado y que es intrínseco al propio principio. Incluso más, en varias oportunidades, los principios parecen no especificar tampoco cómo se llega al estado de cosas o bien que los realiza. De este modo, parecen solamente estipular la obligatoriedad de utilizar medios idóneos para lograr un determinado fin, aunque sin especificar el modelo de conducta prescripto ${ }^{34}$.

Por lo anterior, como explica JOHN FINNIS, para aplicar este tipo de normas en muchos casos resulta necesario especificar o determinar de una forma racional su contenido. Este proceso implica especificar, al menos: i) la identidad del titular (o titulares) del deber, quien debe respetar o hacer efectivo el derecho enunciado como principio (Pfmd); ii) el contenido del deber o la descripción de los actos para el cumplimiento del deber (incluyendo plazos, circunstancias y condiciones para dicho cumplimiento); iii) la identidad del titular del derecho o la descripción de su clase; iv) las condiciones por las que se puede perder el derecho e, incluso, si su titular puede renunciar a él y en qué condiciones; y v) los límites de las libertades del titular del derecho ${ }^{35}$.

La importancia de la determinación o concreción judicial en la aplicación de los principios (tanto por su especificación como adecuados para resolver determinados tipos de casos, como por las consecuencias y soluciones jurídicas que de ellos se extraen) se refleja en el valor y la utilidad que tiene el precedente judicial en relación con cómo aplicarlos. El valor y el interés que los operadores jurídicos les dan a estos precedentes no es tanto por la respuesta individual que se ha ofrecido a un caso, sino, más bien, la delimitación que se hace de la norma general que se aplicó al caso ${ }^{36}$.

Estas dificultades en la aplicación de muchos de los principios, tanto para integrar la interpretación de otra norma como para resolver un caso, parecen haber sido reconocidas por los principales defensores de la distinción entre principios y reglas (incluso por el propio DWORKIN) al advertir que su aplicación no es "disyuntiva" o "a todo o nada" como la de las reglas. La aplicación de los principios admite grados de cumplimiento ${ }^{37}$ y, por ello, cuando en un caso dos principios entran en conflicto, el encargado de dirimirlo ha de tener en cuenta el "peso relativo" de cada uno de ellos ${ }^{38}$. Esto lo admite incluso ROBERT ALEXY, que caracteriza los principios como mandatos de optimizacin que ordenan que algo debe ser realizado en la mayor medida de lo posible, dentro de las posibilidades jurídicas y reales existentes ${ }^{39}$. De hecho, debido a lo indeterminados que resultan los principios en muchos casos, ALEXY considera que no hay (ni sería razonable que hubiese) sistemas jurídicos solo compuestos de principios. A su juicio, "un sistema de principios puro, debido a su indeterminación y debilidad, contradiría exigencias irrenunciables de la seguridad jurídica” ${ }^{40}$. Por ello, considera que los sistemas jurídicos suelen estar compuestos por reglas, principios y procedimientos sobre cómo se crean, modifican y aplican estos principios y reglas ${ }^{41}$.

Si bien (conforme a lo que arriba ha sido advertido) el carácter prima facie de algunos de los principios (es decir, de su apertura a otras razones) requiere que sean determinados al momento de su aplicación, esta necesidad de determinación parece menor en el proceso de decisión acerca de si los tipos de acciones específicas están o no regulados por el contenido de un principio (o si un tipo de interpretación específica es más conforme al contenido de un principio que otros tipos de interpretación) que la necesidad de concreción 
que requieren para pasar de su contenido y de los múltiples posibles modos graduales de respetarlo a una norma de acción singular que establezca cómo se efectivizará la solución jurídica para el caso ${ }^{42}$.

\section{Más de una respuesta justa y el problema de la perplejidad judicial}

Por último, frente al argumento que defiende la existencia de una única respuesta correcta porque, de otro modo, los jueces no podrían resolver, ya que si no hubiera en concreto una alternativa mejor, la voluntad no podría elegir su puesta en la existencia y la acción decisoria se paralizaría, surgen varios interrogantes: ¿cómo se produce entonces la acción en casos en los que el entendimiento ofrece opciones de resolución cuyas razones son relativamente suficientes (y no absoluta o necesariamente suficientes)? ¿Cómo salimos de la indecisión y de la inmovilidad frente a razones insuficientes? ¿Cómo se lleva a cabo una decisión judicial frente a opciones justas prácticamente iguales?

La voluntad (nuestra inclinación a decidir por una de las opciones justas de resolución) no queda necesariamente inmóvil frente a la inconmensurabilidad de los argumentos para elegir entre las opciones razonables. Una de las maneras en que se supera esa inmovilidad de la voluntad para decidir frente a opciones desde el punto de vista de los argumentos como igualmente razonables puede darse "por la disposición del hombre”, por el impulso de la persona ${ }^{43}$. Más específicamente, en estos casos, el querer decidir razonablemente puede, a su vez, querer aprovechar el impulso de las disposiciones afectivas según los hábitos de carácter de la persona que decide para lograr optar entre las distintas posibilidades razonables de la decisión ${ }^{44}$.

Esto no supone defender una visión emotivista o irracionalista de la decisión judicial, por dos razones. En primer lugar, porque quien decide lo hace dentro de un grupo de opciones previamente consideradas razonables. En segundo lugar, hay razones para que la voluntad quiera la intervención de las disposiciones afectivas según los hábitos de carácter de la persona que decide a fin de desbloquear su inmovilidad e inclinar la balanza en los casos en que hay que decidir por una opción entre varias razonables. Las teorías de la decisión judicial basada en las virtudes (virtue jurisprudence) han reflexionado sobre estas razones ${ }^{45}$. Para estas teorías, las disposiciones de carácter para elegir lo que es bueno, noble o justo (virtudes), indiscutidamente ayudan a tomar resoluciones justas para los casos e, incluso, nos ofrecen el mejor criterio para determinar qué decisiones son justas, al punto de que puede defenderse que "lo que determina la corrección de una decisión es que un juez virtuoso podría haber tomado tal decisión” ${ }^{46}$. Así, podría decirse que, en general, las virtudes juegan un papel constitutivo de las decisiones judiciales justas y, en particular, de aquellas en las que parece haber más de una decisión justa posible ${ }^{47}$. Esto no supone afirmar que solo un juez virtuoso puede tomar decisiones justas, sino que quien no lo sea también puede hacerlo aprovechando o decidiendo con los mismos criterios de otro que sí lo es ${ }^{48}$. En último término, esto sería así porque la mejor acción siempre será aquella que logre integrar del mejor modo posible el complejo de facultades corpóreo-anímicas (razón, voluntad, inclinaciones sensibles) de quien decide "de manera que las tendencias mismas de los sentidos lleguen a ser un principio del actuar humano configurado por la razón" ${ }^{49}$. Esto se logra por medio del hábito de integrar estas dimensiones en el obrar, lo que genera "una especie de disposición estable o de inclinación adquirida que permite realizarlos con la corrección correspondiente a cada facultad en cuestión: con facilidad, espontaneidad y buen tino" 50 .

Ahora bien, ¿`sostener que los hábitos afectivos razonables nos ayudan a elegir una de las diferentes opciones justas y que hay razones para seguir estas inclinaciones no supone, en realidad, defender una versión especial de "la tesis de la respuesta más justa"? Esto no necesariamente es así por dos razones. En primer lugar, si bien hay razones para complementar los elementos racionales y argumentables de una decisión con inclinaciones afectivas razonables, no siempre estos elementos afectivos se pueden incorporar a la justificación de la decisión, pues no logran brindar razones públicas que hagan de la opción elegida la más justa y que excluyan el resto de opciones como menos justas o injustas ${ }^{51}$. Esto, por un lado, supone que sea razonable esperar que diferentes 
jueces con distintas disposiciones de carácter opten para un mismo caso por diferentes posibilidades justas de respuesta. Por el otro, no supone que las decisiones en las que se debe optar por una resolución entre varias posibles justas no puedan ser debidamente justificadas. En realidad, todas las opciones justas de resolución de estos casos pueden justificarse en las razones que hacen que esa opción justa sea razonable.

En segundo lugar, tener en cuenta los hábitos afectivos no elimina totalmente la posibilidad de que tenga que decidirse un caso entre opciones igualmente justas, porque pueden darse casos en que los operadores jurídicos (obligados a decidir) estén perplejos frente a dos o más posibilidades justas de decisión, incluso desde la perspectiva que les proporcionan sus inclinaciones de carácter. En estos casos, por el impulso de la voluntad, quien decide puede considerar por medio de su entendimiento especialmente algo bajo determinada perspectiva y no bajo otra que le permita tomar una decisión. Esto supone aceptar que en estos casos la causa (una razón) de que la voluntad quiera algo no siempre actúa con un carácter necesario, "porque la voluntad misma puede presentar un impedimento, o bien eliminar esa consideración que la induce a querer, o bien considerando lo opuesto" ${ }^{52}$. Como explica CARLOS LLANO-CIFUENTES, al final de todas las consideraciones hay un reducto inabordable en que la voluntad de la persona es todavía dueña de su decisión y no puede ser al fin y al cabo conducida por nadie ${ }^{53}$. En estos casos, hay algo que la voluntad aplica a una opción más que a las otras. Esta decisión "que opta por un bien con preferencia sobre otro igual, no es estrictamente irracional, ya que hay un bien adicional en el uso de su propia libertad, y ello es mejor que suspender la decisión por no haber motivo real preferente para ella, o por no haber podido encontrarlo; peor sería quedar perplejos en la duda y en la inacción. No es irracional, ni irresponsable: cuanto más conscientes de los motivos que verdaderamente han entrado en juego dentro de la decisión, más libres y responsables nos hacemos respecto de ella; peor sería creer que hemos decidido con objetividad, cargando sobre una supuesta bondad objetiva (inexistente) el peso de la motivación, y ocultar el subyacente motivo de nuestra propia libertad, que es quien ha inclinado en verdad nuestra decisión" ${ }^{54}$.

\section{Conclusiones}

Por más que hoy resulte bastante claro que el conjunto de leyes dictadas por el legislador no logra ofrecer un único resultado correcto para cada controversia jurídica, algunos autores, partiendo de la distinción entre el plano de la corrección formal de las decisiones judiciales y el de su justicia, defienden que el derecho en su conjunto es capaz de ofrecer una única resolución justa para cada caso. Los principales argumentos para defender esto son tres.

Por un lado, se advierte que si bien es comprensible que toda interpretación abstracta (o dogmática) de una norma conduzca a varios resultados posibles, no sucede lo mismo cuando la interpretación se realiza para la resolución de un caso en concreto. Esto sería así porque de no conducir a un único resultado mejor, la voluntad no podría elegir su puesta en la existencia y la acción decisoria se paralizaría. Además, por la específica finalidad que persigue la interpretación en concreto, esta particular finalidad hace que el sistema jurídico simplifique la interpretación en concreto al presentarla como bivalente. Por último, el argumento que parece tener mayor peso para defender "la tesis de la respuesta más justa" advierte que frente a las múltiples posibilidades interpretativas de una norma para resolver un caso en concreto, ha de decidirse por aquella que mejor realiza el conjunto de principios que justifican el derecho en general y la decisión en particular. Este argumento ha cobrado especial relevancia desde que muchos de estos principios están expresamente recogidos por las prácticas constitucionales, legales y jurisprudenciales de los sistemas jurídicos occidentales.

Si bien consideramos aceptable la distinción entre el plano de la corrección formal y el de la justicia, con este trabajo se busca mostrar que los tres principales argumentos para defender la "tesis de la respuesta más justa" (argumento de que el máximo grado de concreción exige una única respuesta, de la bivalencia jurídica y de los principios) no resultan capaces de lograr su objetivo. Por más que la finalidad de la interpretación 
concreta limita las posibilidades interpretativas, no asegura un único resultado. Además, frente a los casos que admiten más de un modo justo de resolución, la voluntad no queda necesariamente inmóvil. Una de las maneras en que se supera esa inmovilidad de la voluntad para decidir frente a opciones desde el punto de vista de los argumentos como igualmente razonables puede darse por la disposición razonable de quien decide. A su vez, la técnica de la bivalencia jurídica tampoco logra completamente su objetivo, ya que la propia práctica muestra que inevitablemente van surgiendo categorías intermedias para resolver mejor los casos concretos. Por último, aunque los principios jurídicos sirvan para orientar la decisión judicial e, incluso, la interpretación de otras normas, no logran necesariamente conducir a un único resultado, entre otras cosas porque una de las características con las que se suelen identificar algunos de estos principios es que están intrínsecamente necesitados de concreción o determinación porque suelen admitir más de un modo similar de ser desarrollados o efectivizados.

Igualmente, consideramos que estos argumentos sí son capaces de mostrar que el derecho logra con diferente intensidad acotar los márgenes de decisión judicial según en qué etapa de esta decisión nos encontremos. Así, por ejemplo, el propio sistema jurídico y la práctica que de él hacen los operadores jurídicos parecen reconocer un mayor margen de maniobra para los jueces y, por tanto, una relajación de la pretensión de la bivalencia de la respuesta jurídica en los elementos de la decisión que resuelven no tanto juzgando las acciones realizadas en el pasado, sino decidiendo hacia el futuro sobre las consecuencias o soluciones concretas que les corresponden a estas acciones. A su vez, si bien el carácter prima facie de algunos de los principios requiere que sean determinados al momento de su aplicación, esta necesidad de determinación parece menor en el proceso de decisión acerca de si un tipo específico de acción está o no regulado por el contenido de un principio, que la necesidad de concreción que requieren para pasar de su contenido y de los múltiples posibles modos graduales de respetarlo a una norma de acción singular que establezca cómo se efectivizará la solución jurídica para el caso.

\section{Bibliografía}

\section{Libros}

ALEXY, ROBERT, El concepto y la validez del derecho (JORGE M. SEÑA, trad., Gedisa, Barcelona, 1997). ALEXY, ROBERT, Teoria de los derechos fundamentales (ERNESTO GARZÓN-VALDÉS, trad., Centro de Estudios Políticos y Constitucionales, Madrid, 1993).

AMAYA-NAVARRO, MARÍA AMALIA, Virtudes judiciales y argumentación. Una aproximación a la ética jurídica (Colección Temas selectos de Derecho Electoral 6, Tribunal Electoral del Poder Judicial de la Federación, México, 2009). Disponible en: http://www.filosoficas.unam.mx/ amaya/publicaciones/06_virtudes.pdf

AQUINO, TOMÁS DE, De malo.

DWORKIN, RONALD, A Matter of Principle (Harvard University Press, Cambridge, Massachusetts, 1985).

DWORKIN, RONALD, Is there Really No Right Answer in Hard Cases?, en A Matter of Principles, 119-145 (Harvard University Press, Cambridge, Massachusetts, 1985).

DWORKIN, RONALD, La justicia con toga (MARISA IGLESIAS-VILA, trad., ÍNIGO ORTIZ DE URBINAGIMENO, ed., Marcial Pons, Madrid, 2007).

DWORKIN, RONALD, La justicia para erizos (HORACIO PONS, trad., Fondo de Cultura Económica, FCE, Buenos Aires, 2014).

DWORKIN, RONALD, Law's Empire (Harvard University Press, Cambridge, Massachusetts, 1986).

DWORKIN, RONALD, Taking Rights Seriously (Duckworth, London, 1979).

ENDICOTT, TIMOTHY A. O., Vagueness in Law (Oxford University Press, Oxford, 2000).

ETCHEVERRY, JUAN BAUTISTA, ¿De qué hablamos cuando hablamos de principios? Una aproximación a los principios jurídicos desde una perspectiva funcional (Pro-manuscrito, Buenos Aires, 2018). 
FINNIS, JOHN, Commensuration and Public Reason, en Reason in Action. Collected Essays, Volume I, 233-255 (Oxford University Press, Oxford, 2011).

FINNIS, JOHN, Ley natural y derechos naturales (CRISTÓBAL ORREGO S., trad., Abeledo Perrot, Buenos Aires, 2000).

GARCÍA-FIGUEROA, ALFONSO, Principios y positivismo jurídico (Centro de Estudios Políticos y Constitucionales, CEPC, Madrid, 1998).

HART, HERBERT. L. A., El concepto de derecho (GENARO R. CARRIÓ, trad., Abeledo Perrot, Buenos Aires, 1963).

LLANO-CIFUENTES, CARLOS, Diferencia entre bien y motivo y su secuela para la práctica, en Examen filosófico del acto de la decisión, 49-138 (Eunsa, Pamplona, 2010).

LLANO-CIFUENTES, CARLOS, La decisión humana ante bienes prácticamente iguales, en Examen filosófico del acto de la decisión, 11-48 (Eunsa, Pamplona, 2010).

MASSINI-CORREAS, CARLOS IGNACIO, Dworkin, Finnis y la "única respuesta correcta", en Objetividad jurídica e interpretación del Derecho, 143-166 (Porrúa, Universidad Panamericana, México, 2008).

RAZ, JOSEPH, The Authority of Law: Essays on Law and Morality (The Clarendon Press, Oxford University Press, New York, Oxford, 1979).

RHONHEIMER, MARTIN, La perspectiva de la moral. Fundamentos de la ética filosófica (JOSÉ CARLOS MARDOMINGO SIERRA, trad., Rialp, Madrid, 2000).

\section{Colaboración en obras colectivas}

POUND, ROSCOE, Mi filosofía del derecho, en El ámbito de lo jurídico. Lecturas de pensamiento jurídico contemporáneo, 303-314 (POMPEU CASANOVAS \& JOSEP JOAN MORESO, eds., POMPEU CASANOVAS, trad., Crítica, Barcelona, 1994).

ZAMBRANO, PILAR, Interpretar es conocer. Una defensa de la teoría referencialista de la interpretación, en Filosofía práctica y derecho. Estudios sobre teoria jurídica contemporánea a partir de las ideas de Carlos Ignacio MassiniCorreas, 53-74 (JUAN CIANCIARDO, JUAN B. ETCHEVERRY, CARLOS DIEGO MARTÍNEZCINCA, PEDRO RIVAS, JAVIER SALDAÑA-SERRANO \& PILAR ZAMBRANO, coords., Universidad Nacional Autónoma de México, Instituto de Investigaciones Jurídicas, UNAM México, 2016).

\section{Revistas}

AARNIO, AULIS, La tesis de la única respuesta correcta y el principio regulativo del razonamiento jurídico, 8 Doxa: Cuadernos de Filosofia del Derecho, 23-38 (1990). http://dx.doi.org/10.14198/DOXA1990.8.01. Disponible en: https://rua.ua.es/dspace/bitstream/10045/10797/1/Doxa8_01.pdf

ATIENZA, MANUEL, Sobre la única respuesta correcta, 6 Juridicas, 2, 13-26 (2009). Disponible en: https://www.r edalyc.org/pdf/1290/129012568002.pdf

ATIENZA, MANUEL \& RUIZ-MANERO, JUAN, Sobre principios y reglas, 10 Doxa: Cuadernos de Filosofia del Derecho, 101-120 (1991). DOI: https://doi.org/10.14198/DOXA1991.10.04. Disponible en: https://rua.ua.e s/dspace/bitstream/10045/10763/1/doxa10_04.pdf

CIANCIARDO, JUAN, Principios y reglas: una aproximación desde los criterios de distinción, 36 Boletín Mexicano de Derecho Comparado, 108, 891-906 (2003). Disponible en: http://www.ejournal.unam.mx/bmd/bolmex108/ BMD10804.pdf

DWORKIN, RONALD, La justicia para erizos a debate, 94 Lecciones y Ensayos, 347-371 (2015). Disponible en: htt p://www.derecho.uba.ar/publicaciones/lye/revistas/94/la-justicia-para-erizos-a-debate.pdf

ETCHEVERRY, JUAN BAUTISTA, Discrecionalidad judicial. Causas, naturaleza y limites, 15 Teoria y Derecho: Revista de Pensamiento Jurídico, 148-171 (2014). 
ETCHEVERRY, JUAN BAUTISTA, Objetividad y determinación del Derecho. Un diálogo con los herederos de Hart (Comares, Granada, 2009).

GARCÍA-AMADO, JUAN ANTONIO, ¿Existe la discreción en la decisión judicial?, 35 Isegoría, Revista de Filosofía Moral y Politica, 151-172 (2006). Disponible en: http://isegoria.revistas.csic.es/index.php/isegoria/article/vie $\mathrm{w} / 34 / 34$

GUASTINI, RICCARDO, Principios de derecho y discrecionalidad judicial, 34 Juecespara la democracia, 39-46 (1999). Disponible en: https://dialnet.unirioja.es/servlet/articulo?codigo $=174776$

HOFFMASTER, BARRY, Understanding Judicial Discretion, 1 Law and Philosophy, 1, 21-55 (1982).

MOORE, MICHAEL S., Four Reflections on Law and Morality, 48 William and Mary Law Review, 5, 1523-1569 (2007). Disponible en: https://scholarship.law.wm.edu/cgi/viewcontent.cgi?article $=1185 \&$ context $=w m l r$

SOLUM, LAWRENCE B., Virtue jurisprudence: una teoría de la decisión judicial centrada en las virtudes, 69 Persona y Derecho, 5-51 (2013). Disponible en: https://www.unav.edu/publicaciones/revistas/index.php/persona-y-de recho/article/view/1571/1442

VIGO, RODOLFO LUIS, Razonamiento justificatorio judicial, 21 Doxa: Cuadernos de Filosofia del Derecho, 483-499 (1998). Disponible en: https://rua.ua.es/dspace/bitstream/10045/10377/1/doxa21-2_33.pdf

\section{Jurisprudencia argentina}

Cámara Nacional de Apelaciones en lo Civil, Sala H, 16 de diciembre de 2002 sobre Fundación Mujeres en Igualdady otro c/Freddo SA s/amparo. http://www.derechoshumanos.unlp.edu.ar/assets/files/documentos/fallo-fundacio n-mujeres-en-igualdad-y-otros-contra-freddo-sa-sobre-amparo.pdf

\section{Notas}

\section{* Artículo de investigación}

1 JUAN ANTONIO GARCÍA-AMADO sostiene que las posiciones formalistas que niegan la discrecionalidad judicial han evolucionado desde las antiguas escuelas de la exégesis y de la jurisprudencia de conceptos del siglo XIX hacia las modernas concepciones del derecho como integridad (de RONALD DWORKIN) o las posiciones neoconstitucionalistas. Cfr. JUAN ANTONIO GARCÍA-AMADO, ¿Existe la discreción en la decisión judicial?, 35 Isegoria, Revista de Filosofia Moral y Politica, 151-172, 151 ss. (2006).

2 Algunos autores prefieren identificar todo el problema de la indeterminación del Derecho con el concepto de laguna. Por ejemplo, JOSEPH RAZ propone una definición amplia de laguna. En concreto, sugiere que hay una laguna jurídica cuando algunas cuestiones jurídicas sujetas a la jurisdicción competente no tienen una respuesta completa. Como puede observarse, este concepto parece incorporar toda causa de indeterminación jurídica. Cfr. JOSEPH RAZ, The Authority of Law: Essays on Law and Morality, 53-77 (The Clarendon Press, Oxford University Press, New York, Oxford, 1979).

3 Cfr. MICHAEL S. MOORE, Four Reflections on Law and Morality, 48 William and Mary Law Review, 5, 1523-1569, 1529-1531 (2007).

4 Cfr. HERBERT. L. A. HART, El concepto de derecho, 157-159 (GENARO R. CARRIÓ, trad., Abeledo Perrot, Buenos Aires, 1963).

5 Sobre las dificultades para evitar toda posible indeterminación del Derecho apelando al référé legislatif, a la negación lógica de las lagunas, a criterios legales que resuelvan las antinomias, a la intención de los legisladores, al propósito de la norma, etc., cfr. JUAN BAUTISTA ETCHEVERRY, Discrecionalidad judicial. Causas, naturaleza y limites, 15 Teoria y Derecho: Revista de Pensamiento Jurídico, 148-171, 151-152 (2014).

6 Junto con estos tres argumentos que defienden la existencia de una única respuesta correcta o justa para cada caso, suele rechazarse la discrecionalidad judicial con, al menos, tres argumentos. El primero de los argumentos es que el escepticismo que dice "no existe en verdad una respuesta correcta" porque hay muchas opiniones al respecto no es capaz de diferenciar entre la incertidumbre y la indeterminación jurídica. La incertidumbre es aquella por la cual desconozco cuál es la respuesta correcta. 
La indeterminación es la falta de una respuesta correcta. Para pasar de la incertidumbre sobre cuál es la respuesta correcta a la indeterminación se necesita un argumento que explique por qué la mejor interpretación del derecho es que se encuentra indeterminado. Este argumento no suele ser ofrecido por los escépticos. Cfr. RONALD DWORKIN, La justicia para erizos, 65 y 119 ss. (HORACIO PONS, trad., Fondo de Cultura Económica, FCE, Buenos Aires, 2014) y más claramente en RONALD DWORKIN, La justicia para erizos a debate, 94 Lecciones y Ensayos, 347-371, 354-355 (2015). El segundo de ellos se basa en la falta de legitimidad democrática de los jueces para crear derecho, ya que no son elegidos por los ciudadanos y, por lo tanto, no son responsables frente a ellos como lo es el legislador ante el electorado. El tercero de estos argumentos se funda en que la creación judicial de derecho sería un grave atentado al principio de irretroactividad de las leyes. Cfr. RONALD DWORKIN, Taking Rights Seriously, 84-86 (Duckworth, London, 1979).Si bien estos argumentos podrían ser importantes para criticar las posiciones escépticas o para justificar la indeseabilidad de la discrecionalidad judicial, no sirven para defender que para todo caso necesariamente hay unanica respuesta correcta o justa. No obstante, la explicación que se ofrecerá en este trabajo sobre el alcance que tienen la pretensión del derecho de ofrecer para todo caso una sola respuesta adecuada y sobre el fenómeno de la discrecionalidad judicial pretende no caer en la crítica antiescéptica y ser de utilidad para responder a los argumentos de la falta de legitimidad de la creación judicial de derecho por considerarla antidemocrática y retroactiva.

7 Por las razones que se expondrán al final de este epígrafe, es más preciso decir que DWORKIN defiende la tesis de la "respuesta más justificada”. Por otra parte, hay que reconocer que en algunas oportunidades DWORKIN parece proponer una versión de la tesis de la respuesta justa que no dice que ella necesariamente tiene que ser nica. Cfr. RONALD DWORKIN, La justicia para erizos, 42 (2014). De hecho, su famosa comparación del derecho con la redacción de una novela encadenada parece reafirmar esta idea. Al respecto, cfr. RONALD DWORKIN, Law's Empire, 238 (Harvard University Press, Cambridge, Massachusetts, 1986). RONALD DWORKIN, La justicia con toga, 52 ss. (MARISA IGLESIAS-VILA, trad., ÍNIGO ORTIZ DE URBINAGIMENO, ed., Marcial Pons, Madrid, 2007). RONALD DWORKIN, La justicia para erizos a debate..., 347-371, 354-355 (2015).

8 Cfr. RONALD DWORKIN, Is there Really No Right Answer in Hard Cases?, en A Matter of Principles, 119-145, 125 (Harvard University Press, Cambridge, Massachusetts, 1985). Esta idea fue desarrollada en RONALD DWORKIN, Taking Rights Seriously, 81, 107-110 (1979).

9 Cfr. RONALD DWORKIN, Law's Empire, 65-68 (1986).

10 Cfr. RONALD DWORKIN, Law's Empire, 151 (1986).

11 DWORKIN desarrolla con detalle cómo todos los derechos y las obligaciones que puede imponer legítimamente una comunidad política se fundan en último término en el principio de la dignidad humana; es decir, en que se trate el destino de cada uno de los miembros de una comunidad "como de igual importancia que los demás y [se] respete[n] sus responsabilidades individuales por su propia vida”. Cfr. DWORKIN, La justicia para erizos, 402 (2014).

12 Cfr. RONALD DWORKIN, Law's Empire, 404-405 (1986).

13 Cfr. RONALD DWORKIN, Law's Empire, 404 ss. (1986). DWORKIN llega incluso a afirmar que, en realidad, la mejor manera de entender el razonamiento moral (en general) es verlo como la interpretación de conceptos morales que forman una compleja red y que pueden ser mejor servidos por una interpretación específica que por otras. RONALD DWORKIN, La justicia para erizos, $132(2014)$.

14 Cfr. MANUEL ATIENZA, Sobre la única respuesta correcta, 6 Jurídicas, 2, 13-26, 23 (2009). Sobre las posiciones de RONALD DWORKIN y JOHN FINNIS, cfr. JUAN BAUTISTA ETCHEVERRY, Objetividad y determinación del Derecho. Un diálogo con los herederos de Hart, 76 (Comares, Granada, 2009).

15 Cfr. RONALD DWORKIN, Is there Really No Right Answer in Hard Cases?, en A Matter of Principles, 119-145, 120 (Harvard University Press, Cambridge, Massachusetts, 1985).

16 Estamos conscientes de que el Código Penal de Argentina zanja esta disputa doctrinaria en su artículo 80 al considerar como otros posibles agravantes del homicidio que conllevan una pena de prisión o reclusión perpetua el ser perpetrado con alevosía (es decir, actuando sin riesgo o buscando que la víctima esté desprevenida o indefensa), ensañamiento u otro procedimiento insidioso (es decir, con engaño para producir el daño a la víctima).

17 PILAR ZAMBRANO, Interpretar es conocer. Una defensa de la teoría referencialista de la interpretación, en Filosofía práctica y derecho. Estudios sobre teoria juridica contemporánea a partir de las ideas de Carlos Ignacio Massini-Correas, 53-74, 61 (JUAN CIANCIARDO, JUAN B. ETCHEVERRY, CARLOS DIEGO MARTÍNEZ-CINCA, PEDRO RIVAS, JAVIER SALDAÑA- 
SERRANO \& PILAR ZAMBRANO, coords., Universidad Nacional Autónoma de México, Instituto de Investigaciones Jurídicas, UNAM México, 2016).

18 CARLOS IGNACIO MASSINI-CORREAS, Dworkin, Finnis y la "unica respuesta correcta", en Objetividad jurídica e interpretación del Derecho, 143-166, 156 (Porrúa, Universidad Panamericana, México, 2008).

19 CARLOS IGNACIO MASSINI-CORREAS, Dworkin, Finnis y la "inica respuesta correcta"..., 143-166, 159 (2008).

20 A juicio de CARLOS IGNACIO MASSINI-CORREAS, "la razón delibera (deliberatio) ante todo acerca de cuáles son las distintas posibles alternativas de solución, analiza cada una de ellas en su naturaleza y sus circunstancias, para decidir luego, movida la razón por la voluntad ordenada al bien, cuál es la solución más justa en el caso concreto. Esta resolución es el objeto del denominado último juicio práctico (iudicium electionis), el cual es seguido por la elección, por parte de la voluntad (intentio), de esa alternativa como la mejor y por la consiguiente dirección de la razón (imperium) a la voluntad para la realización efectiva de la opción elegida (usus)", CARLOS IGNACIO MASSINI-CORREAS, Dworkin, Finnis y la "ínica respuesta correcta”..., 143-166, 157-158 (2008).

21 MANUEL ATIENZA, Sobre la única respuesta correcta..., 13-26, 24 (2009).

22 Cfr. RONALD DWORKIN, Law's Empire, 65 ss., 337 ss. (1986). De hecho, DWORKIN distingue los niveles semánticos, filosóficos, doctrinal y judicial de la interpretación jurídica. A su juicio, el valor de la integridad que le atribuye a la práctica jurídica fluye del doctrinal al de la aplicación judicial. Cfr. RONALD DWORKIN, La justicia con toga, 19-31 (2007).

23 Cfr. AULIS AARNIO, La tesis de la única respuesta correcta y el principio regulativo del razonamiento jurídico, 8 Doxa: Cuadernos de Filosofia del Derecho, 23-38, 23-24 (1990).

24 Sobre los distintos modos de entender la discrecionalidad judicial y, por tanto, la tesis de la única respuesta correcta que niega esa discrecionalidad, cfr. BARRY HOFFMASTER, Understanding Judicial Discretion, 1 Law and Philosophy, 1, 21-55, 27 ss. (1982).

25 Cfr. TIMOTHY A. O. ENDICOTT, Vagueness in Law, $72-73$ (Oxford University Press, Oxford, 2000).

26 Cfr. PILAR ZAMBRANO, Interpretar es conocer. Una defensa de la teoría referencialista de la interpretación..., 53-74, 59 ss. (2016). RODOLFO LUIS VIGO reconoce el importante papel de los jueces a la hora de determinar el derecho y también desarrolla lo que denomina las diferentes "premisas del silogismo judicial". Cfr. RODOLFO LUIS VIGO, Razonamiento justificatoriojudicial, 21 Doxa: Cuadernos de Filosofía del Derecho, 483-499, 492 ss. (1998).

27 Cfr. los artículos 260 y siguientes del Código Civil y Comercial de Argentina.

28 Cfr. JOHN FINNIS, Ley natural y derechos naturales, 306 ss. (CRISTÓBAL ORREGO S., trad., Abeledo Perrot, Buenos Aires, 2000).

29 Hay diferentes criterios para atribuir a un precepto la categoría de principio. Como explica RICCARDO GUASTINI, un modo de hacerlo es afirmando que el rasgo que caracteriza a los principios es su "peculiar estructura lógica". A diferencia de las reglas, los principios serían normas con un supuesto de hecho abierto (no concreto y determinado) y sin una específica consecuencia jurídica. En cambio, otros identifican los principios conforme a la posición peculiar que ocupan en el ordenamiento jurídico (o en uno de sus subsectores). Los principios serían normas "fundamentales, caracterizadoras, que confieren identidad axiológica al ordenamiento (a uno de sus subsectores) y ofrecen justificación a las restantes normas...”. RICCARDO GUASTINI, Principios de derecho y discrecionalidad judicial, 34 Jueces para la democracia, 39-46, 39 ss. (1999). En un sentido parecido, cfr. MANUEL ATIENZA \& JUAN RUIZ-MANERO, Sobre principios y reglas, 10 Doxa: Cuadernos de Filosofia del Derecho, 101-120, 105 ss. (1991). FINNIS parece partir de este último criterio cuando distingue entre principios con fuerza concluyente (que emplean una forma negativa) y los principios con fuerza directiva (que emplean fórmulas asertivas). Cfr. JOHN FINNIS, Ley naturaly derechos naturales, 251 ss. (2000).

30 Cfr. MANUEL ATIENZA \& JUAN RUIZ-MANERO, Sobre principios y reglas..., 101-120, 108 (1991).

31 ROSCOE POUND pone énfasis en este aspecto al sostener que los principios jurídicos "no asignan ninguna consecuencia detallada y definida a un estado o situación de hecho definida y mucho menos detallada". ROSCOE POUND, Mi filosofia del derecho, en El ámbito de lo jurídico. Lecturas de pensamiento jurídico contemporáneo, 303-314, 310 (POMPEU CASANOVAS \& JOSEP JOAN MORESO, eds., POMPEU CASANOVAS, trad., Crítica, Barcelona, 1994). 
32 Fallo de la Cámara Nacional de Apelaciones en lo Civil, Sala H, 16 de diciembre de 2002 sobre Fundacin Mujeres en Igualdad y otro c/Freddo SA s/amparo.

33 Entre otros autores, ALFONSO GARCÍA-FIGUEROA advierte que reconocer la presencia de principios en el sistema jurídico puede servir como argumento contra la idea de que hay para todo caso una única respuesta justa y contra la tesis de la bivalencia jurídica. Cfr. ALFONSO GARCÍA-FIGUEROA, Principios y positivismo jurdico, 287, 301 (Centro de Estudios Políticos y Constitucionales, CEPC, Madrid, 1998).

34 A nuestro juicio, parece que las reglas pretenden ser inderrotables, cerradas o absolutas, ya que su estructura parece aspirar a que su aplicación no admita excepciones no explicitadas; mientras que los principios parecen aceptar ser derrotables, abiertos o no absolutos, porque su aplicación admite excepciones no explicitadas. No obstante, estos criterios de diferenciación entre reglas y principios no son fuertes, debido a que las reglas no son tan absolutas, porque puede haber argumentos basados en principios que pueden pesar contra el resultado al que dan lugar; y, viceversa, algunos tipos de principios pueden conformar estándares jurídicos absolutos o que no exhiben un carácter prima facie (por ejemplo, nada debería pesar en contra de los resultados de la aplicación del principio que establece que " $n a d i e$ ha de ser condenado por un delito sino sobre la base de prueba que conduzca más allá de la duda razonable”). Cfr. JUAN BAUTISTA ETCHEVERRY, ¿De qu hablamos cuando hablamos de principios? Una aproximacin a los principios jurdicos desde una perspectiva funcional (Pro-manuscrito, Buenos Aires, 2018).

35 Cfr. JOHN FINNIS, Ley natural y derechos naturales, 247 (2000).

36 Cfr. RODOLFO LUIS VIGO, Razonamiento justificatorio judicial..., 483-499, 494 (1998).

37 DWORKIN utiliza la distinción entre "derechos abstractos” y "derechos concretos" para sostener que en los casos difíciles en los que hay un conflicto entre derechos abstractos basados en principios, los jueces han de expresar qué derechos concretos tienen las partes. Cfr. RONALD DWORKIN, Taking Rights Seriously, 92 ss. (1979). Si bien hay que reconocerle a DWORKIN que la gradualidad que se predica de la aplicación de los principios es diferente, si se los considera en abstracto o en un contexto concreto al que han de ser aplicados. No obstante, esto no niega completamente dicha gradualidad.

38 RONALD DWORKIN, Taking Rights Seriously, 24-26 (1979). Sobre la distinción entre principios y reglas, cfr., entre otros, JUAN CIANCIARDO, Principios y reglas: una aproximacin desde los criterios de distincin, 36 Boletn Mexicano de Derecho Comparado, 108, 891-906 (2003).

39 Cfr. ROBERT ALEXY, Teora de los derechos fundamentales, 81-98 (ERNESTO GARZÓN-VALDÉS, trad., Centro de Estudios Políticos y Constitucionales, Madrid, 1993). Sin embargo, la gradualidad de los principios puede ser puesta en duda, si se considera que la noción de óptimo no puede ser de cumplimiento gradual: el óptimo se alcanza o no se alcanza. Por ello, a fin de que los principios no terminen siendo indistinguibles de las reglas, algunos autores prefieren sugerir que la característica diferenciadora de los principios es su contenido de "deber ser ideal" (ideales Sollen). Este tipo de deber ser no presupone que lo debido sea posible jurídica y fácticamente en su totalidad. Cfr. ALFONSO GARCÍA-FIGUEROA, Principios y positivismo jurdico, 192 ss. (1998).

40 Cfr. ROBERT ALEXY, El concepto y la validez del derecho, 168 (JORGE M. SEÑA, trad., Gedisa, Barcelona, 1997).

41 Cfr. ROBERT ALEXY, El concepto y la validez del derecho, 168, 173-174 (1997).

42 DWORKIN pone énfasis en la importancia de que toda interpretación se ajuste a decisiones y prácticas del pasado para poder justificar hacia adelante lo que se resuelve. Esto puede advertirse en su defensa de los argumentos de los principios y de la bivalencia jurídica desarrollados anteriormente. En La justicia con toga vuelve sobre esta idea al desarrollar el valor de la legalidad. Cfr. RONALD DWORKIN, La justicia con toga, 186 ss. (2007).

43 Cfr. CARLOS LLANO-CIFUENTES, La decisiómbumana ante bienes prácticamentiguales, en Examen filosóficolel acto de la decisin, 11-48, 47 ss. (Eunsa, Pamplona, 2010).

44 JOHN FINNIS le reconoce un papel a la afectividad en la toma de decisiones entre opciones inconmensurables. Expresamente reconoce que esto puede suceder en el ámbito político e, incluso, en el judicial. Cfr. JOHN FINNIS, Commensuration and Public Reason, en Reason in Action. Collected Essays, Volume I, 233-255, 252-254 (Oxford University Press, Oxford, 2011).

45 Cfr. LAWRENCE B. SOLUM, Virtue jurisprudence: una teora de la decisin judicial centrada en las virtudes, 69 Persona y Derecho, 5-51 (2013).

46 Cfr. MARÍA AMALIA AMAYA-NAVARRO, Virtudes judiciales y argumentacin. Una aproximacin a la tica jurdica, 35 (Colección Temas selectos de Derecho Electoral 6, Tribunal Electoral del Poder Judicial de la Federación, México, 2009). 
47 Cfr. MARÍA AMALIA AMAYA-NAVARRO, Virtudes judiciales y argumentación..., 38-46 (2009).

48 De hecho, está en camino de ser virtuoso (específicamente, prudente) el juez que es consciente de que debido a que las acciones contingentes sobre las que decide son tan variadas, se vuelve casi indispensable recurrir a la experiencia que se adquiere a lo largo del tiempo por medio de la memoria personal y el consejo de quienes gocen de mayor experiencia. Cfr. JUAN BAUTISTA ETCHEVERRY, Discrecionalidad judicial. Causas, naturaleza y limites..., 148-171, 162 (2014). Con esta propuesta se busca ofrecer una respuesta sobre el papel de las virtudes en las decisiones judiciales justas entre la visión que considera que solo un juez justo puede tomar decisiones justas (LAWRENCE B. SOLUM) y la que considera que las decisiones justas son las que hubiera tomado un juez justo (MARÍA AMALIA AMAYA-NAVARRO).

49 Cfr. MARTIN RHONHEIMER, La perspectiva de la moral. Fundamentos de la ética filosófica, 204-205 (JOSÉ CARLOS MARDOMINGO SIERRA, trad., Rialp, Madrid, 2000).

50 MARTIN RHONHEIMER, La perspectiva de la moral..., 203 (2000).

51 Sobre este punto, en parte, disentimos de AMAYA. A su juicio, "una concepción de la justificación jurídica basada en las virtudes, no tiene por qué asumir que la sensibilidad perceptual del juez virtuoso impide que este dé razones a favor de su decisión. Por el contrario, el juez virtuoso tiene una sensibilidad especial que, justamente, le permite discernir las razones relevantes que apoyan una determinada decisión en el caso concreto y, desde luego, expresarlas en su sentencia”. Si bien compartimos que un juez virtuoso es capaz de identificar mejor las razones a favor de una decisión, en particular en lo que respecta a las decisiones entre más de una opción justa, la sensibilidad perceptual del juez virtuoso que puede ayudarlo a decidirse con base en razones por una de las opciones justas en parte puede no resultar comunicable en lo que se refiere a la exclusión de las otras opciones por las que no se ha optado. Cfr. MARÍA AMALIA AMAYA-NAVARRO, Virtudes judiciales y argumentación..., $36-37$ (2009).

52 TOMÁS DE AQUINO, De malo, q. 6, na.1, ad. 15.

53 Cfr. CARLOS LLANO-CIFUENTES, La decisión humana ante bienesprácticamente iguales..., 11-48, 48 (Eunsa, Pamplona, 2010).

54 CARLOS LLANO-CIFUENTES, Diferencia entre bien y motivo y su secuela para la práctica, en Examen filosófico del acto de la decisión, 49-138, 57 (Eunsa, Pamplona, 2010).

\section{Licencia Creative Commons CC BY 4.0}

Para citar este artículo / To cite this article: ETCHEVERRY, JUAN BAUTISTA, De la respuesta correcta a la respuesta más justa. La intensidad de la tesis de la respuesta justa en las distintas etapas de la decisión judicial, 138 Vniversitas (2019). https://doi.org/10.11144/Javeriana.vj138.drcr 